"This is the most direct evidence for a collapse of a Fermi volume in any quantum critical matter," said Steglich. "We expect this new insight to have broad implications for other strongly correlated electron systems."

\section{Zigzag-Shaped Magnetic Films Used for Single-Axis Field Sensing}

F.C.S. da Silva and co-workers at the National Institute of Standards and Technology (NIST) in Boulder, Colo., and Gaithersburg, Md., have fabricated and simulated zigzag-shaped magnetic thinfilm elements. They chose the zigzag geometry because it can be used as a single-axis magnetic-field sensor, which is integral to nanoscale devices for data storage technologies. These devices are based on the anisotropic magnetoresistive (AMR) effect. While the AMR effect has a relatively small change in resistance, the devices compensate by their sensitivity to field changes and by very low intrinsic magnetic noise. Both of these properties arise because AMR devices are made from a single layer of magnetic material. Having control over the magnetic easy axis orientation is important for making scalable AMR sensors without complicated current- and field-biasing schemes.

The critical aspect of designing such a device lies in biasing the magnetic and current flows at $45^{\circ}$ to each other to obtain an asymmetric linear response, the researchers said. This is achieved in the zigzag elements by fabricating it in a shape in which the current flows down the center and the shape controls the local magnetic bias. To obtain a uniaxial magnetic element, a soft magnetic material is chosen with both shape and induced anisotropies to ensure that there is just one anisotropy axis.

As reported in the December 13, 2004, issue of Applied Physics Letters (p. 6022; doi: $10.1063 / 1.1834732)$, these structures were fabricated using optical lithography in which a $30 \mathrm{~nm}$ thick $\mathrm{Ni}_{80} \mathrm{Fe}_{20}$ Permalloy film was sputtered onto a $\mathrm{SiO}_{2}$-coated $\mathrm{Si}$ wafer while a field was applied along the element's long axis. Layers of Ta $5 \mathrm{~nm}$ thick were deposited before and after the Permalloy to improve adhesion, provide optimal texture for the film, and protect it from oxidation.

As shown in Figure 1, the magnetic state was imaged using scanning electron microscopy with polarization analysis (SEMPA), which yields high spatial resolution and measures the magnetic direction by analyzing the spin polarization of the secondary electrons emitted during analysis. Additionally, micromagnetic simulations were performed by using the

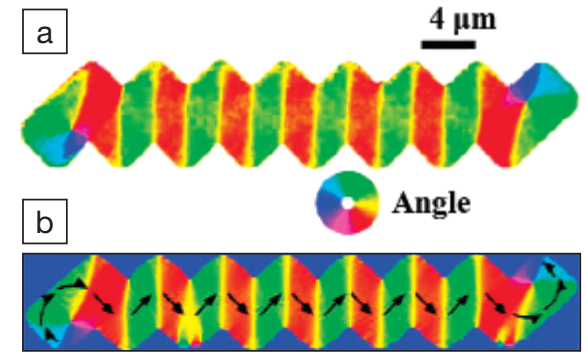

Figure 1. Zigzag-shaped magnetic thinfilm structures. (a) Experimental scanning electron microscopy with polarization analysis image of a zigzag structure. (b) Simulation performed on the same geometry using object-oriented micromagnetic framework software. Reprinted with permission from Applied Physics Letters 85 (24)(2004) p. 6022. (C) 2004 American Institute of Physics.

object-oriented micromagnetic framework (OOMMF) software, a simulation program developed at NIST.

These measurements show that it is possible to naturally bias the magnetization in magnetoresistive sensors by choosing the appropriate contour in the magnetic element. For a single-layer MR sensor, a geometrical biasing mechanism is successful from the nanoscale to the microscale. The researchers said that this technique can be scaled down until the distance between corners becomes comparable to the domain wall width in the magnetic material. SEMPA imaging of fabricated structures along with OOMMF modeling can be used to predict the properties of devices in the nanoscale regime. This type of nanoengineering should have a significant impact in the fields of magnetic sensors and memory applications, the researchers said.

ADITI S. RisBUD

\section{Combustion Spray Process Leads to Rapid Fabrication of Porous SOFC Electrodes}

The fabrication of porous electrodes with high surface area is of great importance for the development of efficient, low-temperature solid-oxide fuel cells (SOFCs). Recently, the focus has been on a combustion chemical vapor deposition (CVD) method that uses only soluble precursors, a relatively new technique that allows the fabrication of porous composite electrodes with nanostructural features and good electrochemical characteristics. Now, scientists at the Georgia Institute of Technology have invented a rapid combustion spray process using a suspension of solid powders with a particle size of less than $1 \mu \mathrm{m}$ in a flammable solvent.
In order to demonstrate the improved performance of SOFC electrodes fabricated by this process, the scientists made and tested combustion spray cathodes deposited onto $\mathrm{Gd}_{0.2} \mathrm{Ce}_{0.8} \mathrm{O}_{2}$ substrates (GDC) supported by a $\mathrm{NiO}(65 \mathrm{wt} \%) / \mathrm{GDC}$ (35 $\mathrm{wt} \%$ ) anode, as reported in the November 2004 issue of the Journal of the American Ceramic Society (p. 2139). The $\mathrm{Sm}_{0.5} \mathrm{Sr}_{0.5} \mathrm{CoO}_{3}$ (SSC) and GDC powders used for the combustion spray step had an average particle size of $0.9 \mu \mathrm{m}$ and $0.3 \mu \mathrm{m}$, respectively, and were suspended in ethanol in a 7:3 weight ratio and ultrasonically dispersed to break up any agglomerates present. A 10 min deposition with a $1250^{\circ} \mathrm{C}$ flame temperature near the sub-

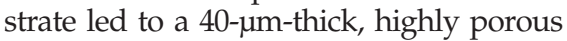
(>40\% porosity) SSC/GDC cathode film. The electrochemical characteristics of the symmetrical cell were determined in ambient air from $450^{\circ} \mathrm{C}$ to $650^{\circ} \mathrm{C}$. The interfacial polarization resistance was only $1.045 \Omega \mathrm{cm}^{2}$ at $500^{\circ} \mathrm{C}$, which is about half of the resistance of, for example, screenprinted systems. Overall, scientists Y. Liu and M. Liu of Georgia Tech's Center for Innovative Fuel Cell Battery Technology found that the performance of the SOFCs made by combustion spray was equal to or better than SOFCs made by other methods. The performance advantage increases with lower temperatures, the researchers said. In addition, the activation energy seems to be reduced.

The researchers said that the advantages of their technique over combustion CVD and similar processes include a significant reduction in the cost of raw materials, capital investment, and operating costs, since the precursors can be used in a powder form and the nozzles are simpler. The unlimited mixing capabilities of their approach (which overcomes the solubility limits of current processes), combined with the availability of the raw materials, allows easy process control, great flexibility in layer composition, and facile formation of multilayer structures. The use of solid particles, rather than expensive organic reagents common for other deposition processes, leads to a significant reduction in materials cost. The open-air operability allows for easy control of the deposition parameters at any stage during the deposition. No high-temperature posttreatment is necessary, and layer thicknesses of $40 \mu \mathrm{m}$ can be formed in minutes.

The developed process is immensely versatile, allowing for the formation of porous as well as dense layers with an almost unlimited variety of compositions, structures, and morphologies, the researchers said.

Alfred A. ZiNN 